\title{
Multidisciplinary Management of Adolescents with Hidradenitis Suppurativa - Series of Cases and Literature Review
}

\author{
Elena Ţarcă, (iD) ${ }^{1,2}$ \\ Elena Cojocaru, (iD ${ }^{2,3}$ \\ Bogdan Caba, 2,4 \\ Alina Costina Luca, ${ }^{2,5}$ \\ Solange Tamara Roșu, 2,6 \\ Viorel Țarcă, 7 Laura Stătescu, ${ }^{8}$ \\ Anca Chiriac, (iD) ${ }^{9}$ \\ Valeriu Vasile Lupu, (iD) 2,5 \\ Eugenia Moroșan, 2,3 \\ Laura Trandafir ${ }^{2,5}$ \\ 'Department of Surgery II-Pediatric Surgery, \\ "Grigore T. Popa" University of Medicine and \\ Pharmacy, lași, 700I I5, Romania; "'Saint \\ Mary" Emergency Children's Hospital, lași, \\ Romania; ${ }^{3}$ Department of Morphofunctional \\ Sciences I - Pathology, "Grigore T. Popa" \\ University of Medicine and Pharmacy, lași, \\ 700I I5, Romania; ${ }^{4}$ Department of \\ Biomedical Sciences, "Grigore T. Popa" \\ University of Medicine and Pharmacy, lași, \\ 700I I5, Romania; ${ }^{5}$ Department of Mother \\ and Child Medicine-Pediatrics, "Grigore \\ T. Popa" University of Medicine and \\ Pharmacy, lași, 700I I5, Romania; \\ "Department of Nursing, "Grigore T. Popa" \\ University of Medicine and Pharmacy, lași, \\ 700I I 5, Romania; ${ }^{7}$ County Statistics \\ Department, lași, 700I I5, Romania; \\ ${ }^{8}$ Department of Dermatology, "Grigore \\ T. Popa" University of Medicine and \\ Pharmacy, lași, 700I I5, Romania; \\ 'Department of Dermatophysiology, \\ Apollonia University, lasi, 700613, Romania
}

Correspondence: Elena Cojocaru; Bogdan Caba

"Grigore T. Popa"University of Medicine and Pharmacy, Universității Street No. 16, lași, 700I 15, Romania

Tel +40765485446

Fax +40232264266

Email elena2.cojocaru@umfiasi.ro; bogdand-caba@umfiasi.ro

\begin{abstract}
Hidradenitis suppurativa (HS) is a recurrent chronic inflammatory condition of the skin with a debilitating potential, especially in adolescents who may experience psychosocial disorders and impaired quality of life. Recognition of typical, recurrent lesions, history of the disease and family investigation establishes the diagnosis. The rarity of the disease in males and the appearance of this condition in children, which is also rare, in two of our adolescent patients, respectively, are the reasons for publishing this article. We reviewed the literature and found that variation exists across international treatment for this HS, and much collaboration is needed to put forth unified and updated recommendations. It is of major importance that the early recognition of the condition and the establishment of treatment in the multidisciplinary team. Psychotherapy, health education, the adoption of a healthy lifestyle, the application of local prevention measures, and long-term medical treatment are components of a correct management. Extensive surgical treatment should be restricted only to severe cases because it may lead to complications, extend the hospitalization period and increase psychological problems in adolescents. The treatment will be personalized, performed according to the stage of evolution, and the approach will be a multidisciplinary one.
\end{abstract} Keywords: hidradenitis suppurativa, children, personalized management, multidisciplinary team

\section{Introduction}

Hurley's disease, hidradenitis suppurativa (HS) or acne inversa is a recurrent chronic inflammatory condition of the follicular epithelium in the axillary region, inguinal, perineal region and sometimes the submammary region. It is a rare disease in children and the recorded literature is correspondingly scarce. ${ }^{1-3}$ In a recent German study, only $4.5 \%$ of all HS patients were children. ${ }^{2}$ HS may be associated with various somatic and psychiatric comorbidities, but data regarding comorbidities in young patients with HS are also sparse. ${ }^{4}$ Due to the recurrent nature, unpleasant odor, associated pain, surgery and subsequent scarring, the disease has a debilitating potential in adolescents who may experience psychosocial disorders and impaired quality of life (QoL). ${ }^{5}$ It is very important that the early recognition of the condition and the establishment of treatment in the multidisciplinary team, as well as psychological support. Because the condition is not very frequent, affecting $1-4 \%$ of the general population, is little known among general practitioners and pediatricians, and the diagnosis can be delayed up to 12 years and over. ${ }^{5-7}$ The predominance is feminine, of $1 / 4$, and the frequent age of appearance is in the second 
or third decade of life. ${ }^{8}$ Although recent hidradenitis suppurativa studies have shown that early-onset disease is associated with a positive family history and more widespread disease, research in pediatric hidradenitis suppurativa is limited and the treatment of HS in children has not been standardized. ${ }^{3,9}$

The rarity of the disease, the appearance of this condition in two male adolescents and the complications that we have to treat were the reasons for publishing this article. We compared the cases treated by us with other cases reported in the literature, and we tried to draw attention to the need for early diagnosis and treatment, as well as the particularities that must be taken into account when adolescents are involved. This article also reviews the therapeutic options for HS in adolescents, highlighting the differences and challenges with treating patients in this age group compared with adults.

\section{Case Report I}

Male patient, FI, 17 years old, smoker, overweight at the time of presentation, from an unfavorable social environment, presents for clinical control of bilateral axillary hidradenitis operated nine months ago, with a hospitalization period of 41 days that time. The bilateral extensive hidradenitis persisted for about a year, was very painful and prevented the patient's physical activity. Because the disease did not respond favorably to topical antiseptic treatment or to Clindamycin antibiotic therapy, surgical excision of necrotic tissues, drainage of purulent collections and dressings with negative pressure was needed. After limiting the infection, the bare areas were quite large and needed to be covered with right axillary advancement flaps and left axillary free skin graft, harvested from the region of the left thigh (Figure 1). The general clinical examination at the second hospitalization found the presence of perineal and scrotal hidradenitis suppurativa in Hurley stage III or severe disease according to IHS4 staging (International HS Severity Score System): the presence of multiple abscesses in different stages of evolution, fistulous trajectories and scar tissue, following a local evolution of 2-3 years (Figure 2).

During a hospitalization period of 3.5 months this time, in our pediatric surgery department of "Saint Mary" Emergency Children Hospital, the patient receives multidisciplinary team treatment, psychotherapy for depression, local and general antibiotic therapy with broad spectrum initially and then according to the culture, isolating Streptococcus anginosus and then Klebsiella pneumoniae

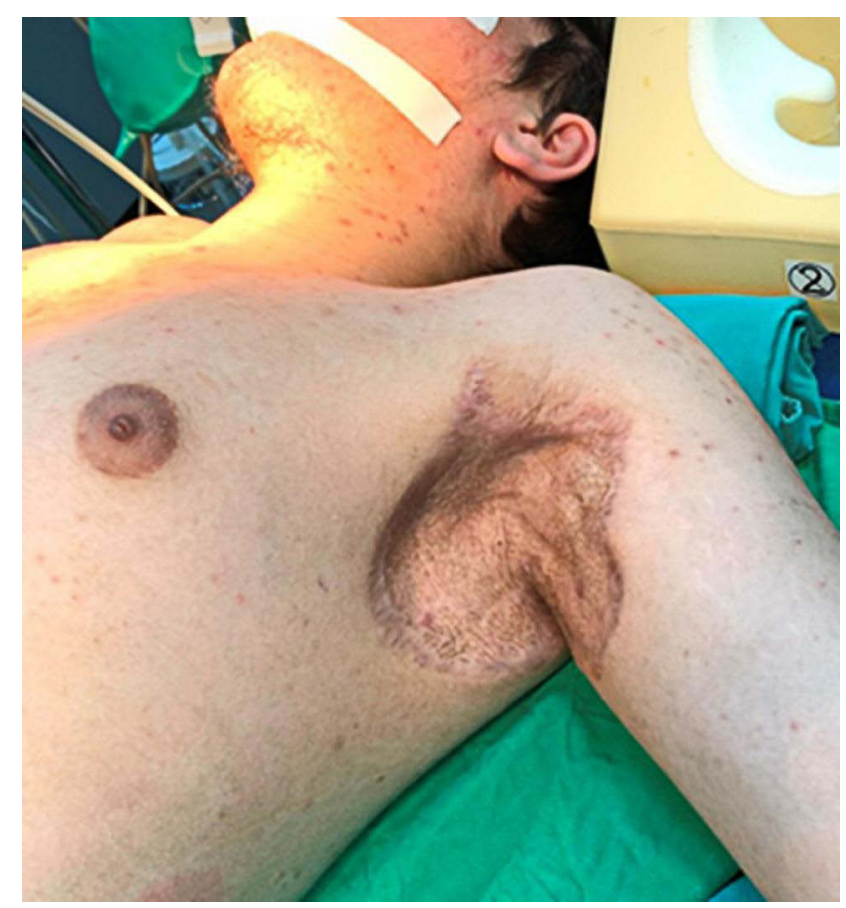

Figure I Excision and grafting of the axillary area, with a favorable evolution after Hurley stage III suppurative hidradenitis.

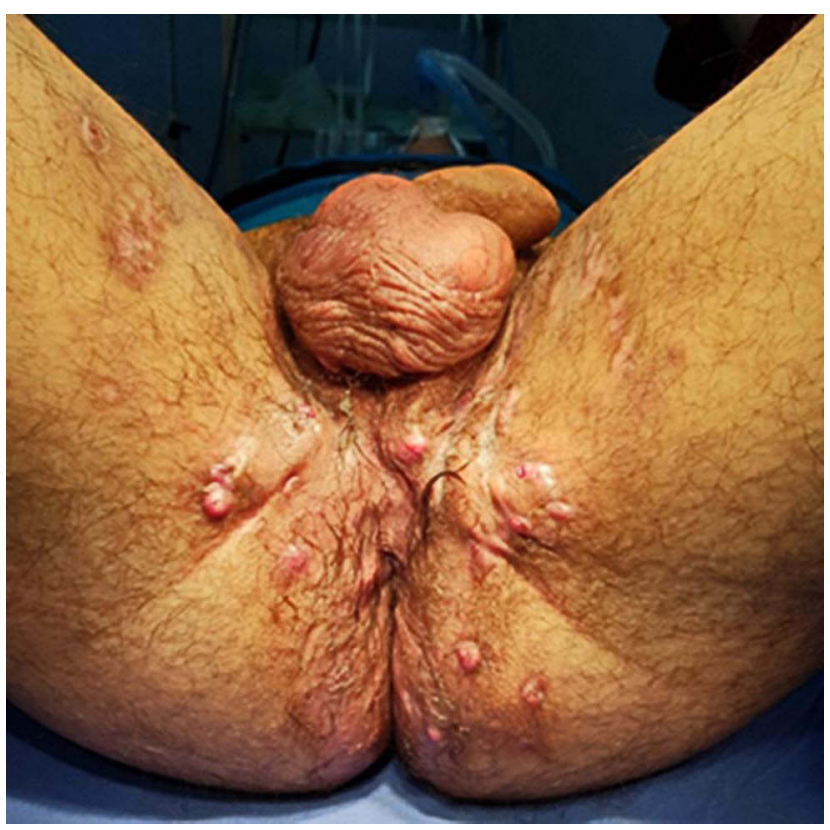

Figure 2 Hurley stage III suppurative hidradenitis, affecting the groin, scrotal, perineal, inner thighs.

MDR from cultures. Because the infection could not be controlled only with antibiotic therapy, the patient undergoes multiple surgeries to evacuate the purulent collections, excision of the infected tissues and fistulas in the perineal and scrotal area; the bare areas remained large and the epithelialization process would have taken a long time, 


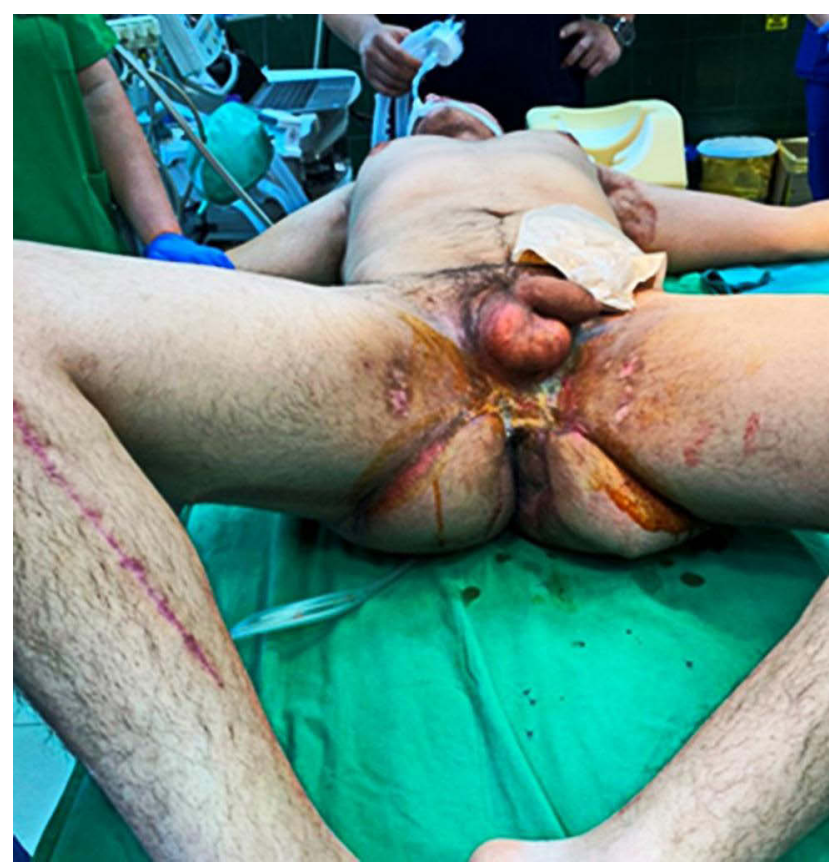

Figure 3 Favorable evolutionary fasciocutaneous flaps under VAC treatment and protection of the perineal area by colostomy. The post-fasciotomy scar is also observed on the right leg.

so we covered the skin defects with two posterior transciocutaneous flaps of transposition at the thigh. The initial postoperative evolution was burdened by the appearance of complications, such as positional compartment syndrome in the right leg, requiring fasciotomy and negative pressure treatment (VAC). The fasciocutaneous flaps show postoperative signs of vascular distress and the areas of skin necrosis are gradually excised, but the subsequent evolution is slowly favorable under VAC treatment and protection of the perineal area by colostomy (Figure 3). During the hospitalization, the excision of the abscesses and of the left sacral, pubic and auricular fistulas was also performed, and the dehiscence of the post-excision sutures at the sacred level is covered with "V-Y" advancement flaps, with favorable evolution. The costs of hospitalization and treatment have reached $15.000 \mathrm{RON}$ for the first hospitalization period and almost 58.000 RON for the second time (15.500 euro in total).

The pathological examination of the excised areas detects skin with reactionary hyperplasia of the epidermis and the presence of dermo-hypodermic fistulous tracts lined with granulation tissue with rich polymorphic inflammatory infiltrate represented by polymorphonuclear, lymphocytes, plasmocytes and multinucleous extracellular giant cells, fibrino-leukocyte on the surface and fibrosis on the wall (Figures 4-6). During the second hospitalization,

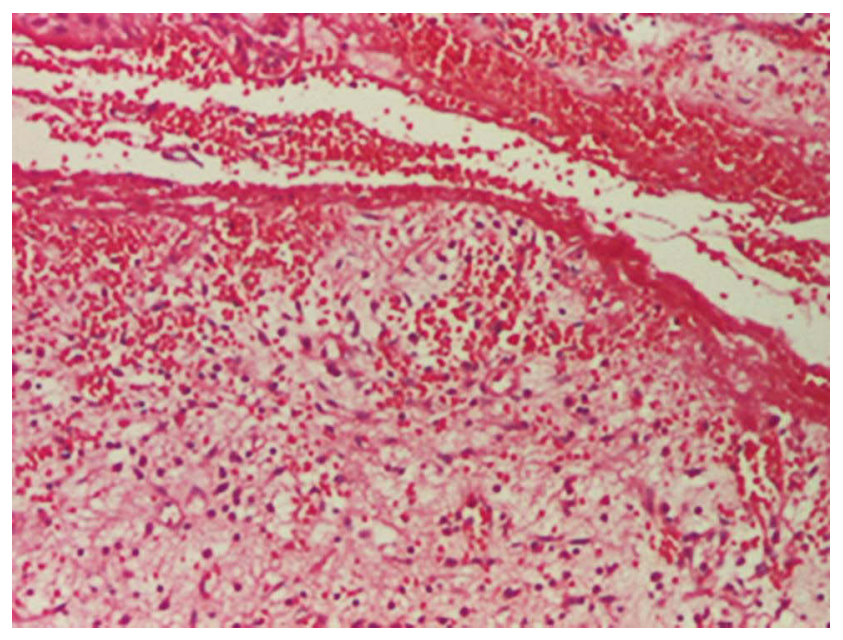

Figure 4 Hyperplasia of the epidermis and the presence of a fistulous tract lined with granulation tissue and hemorrhage (HE stain, $\times 40)$.

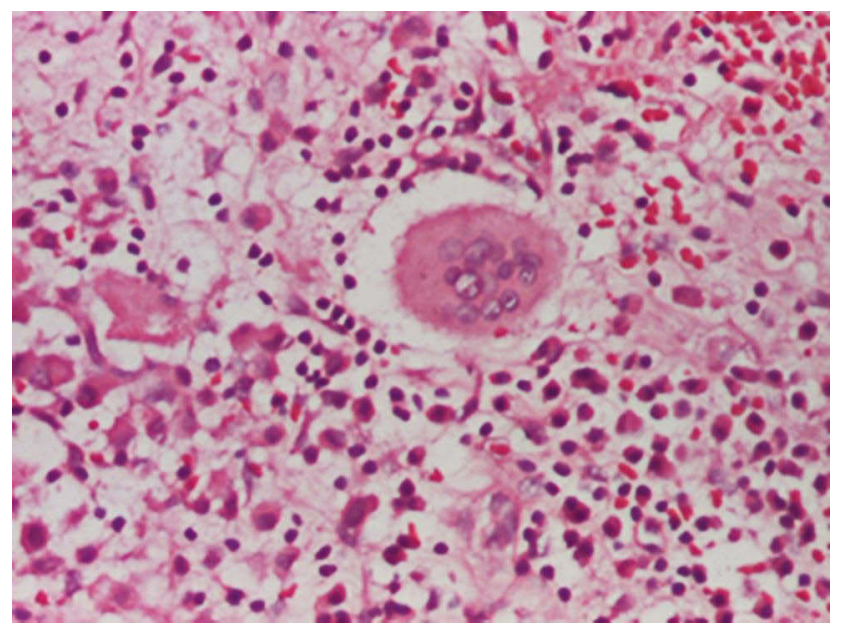

Figure 5 Polymorphic inflammatory infiltrate with polymorphonuclear, lymphocytes, plasmocytes and multinucleous extracellular giant cells (HE stain, x200).

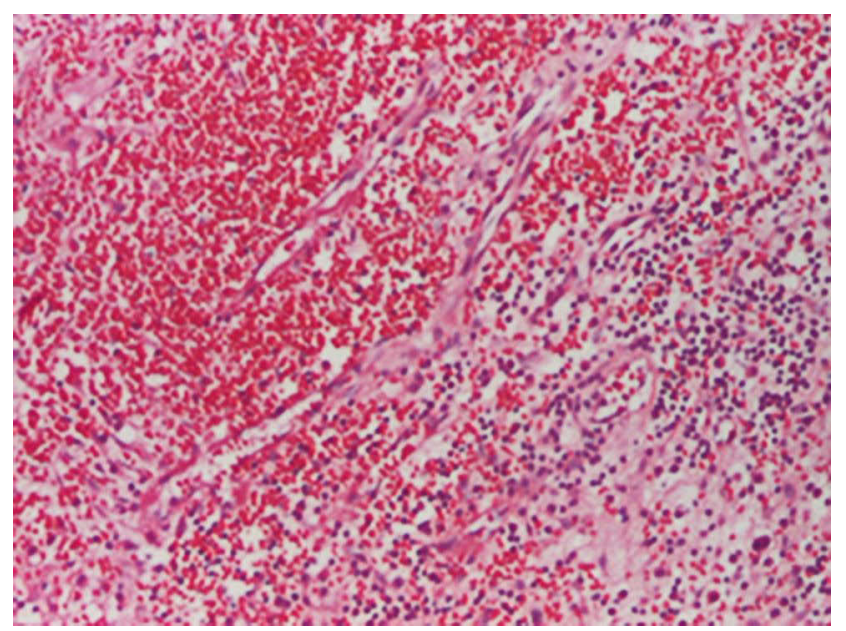

Figure 6 Hemato-fibrino-leukocyte exudate on the surface and fibrosis on the wall (HE stain, $\times 40$ ). 
the patient turned 18 years old and was registered by an adult surgeon to close the colostomy, and by the dermatologist to monitor the evolution of the disease. The parents and the patient's consent were obtained for the publication of photographs and the use of data from the observation file.

\section{Case Report 2}

Male patient, CRT, 17 years old, passive smoker, normal weight, no family history, presents for bilateral axillary hidradenitis, persisting for 3-4 months, treated with clindamycin per os, incisions and drainage made in another hospital unit, but with local recurrences (Figure 7). During clinical examinations at hospitalization, abscesses are also detected at the inguinal and perineal levels (Figure 8), and in addition, the patient associates acne, psoriasis, pectus excavatum, and dextroconvex dorsolumbar scoliosis (Figures 9 and 10). Biological, imaging investigations and multiple clinical consultations performed during hospitalization complete the diagnoses with hypocalcemia, hyposideremia, biological inflammatory syndrome, immunological deficit, anterior mitral valve prolapse, grade I mitral regurgitation, myopia and right L5 spondylolysis (highlighted on lumbar spine computerized tomography). Staphylococcus aureus is

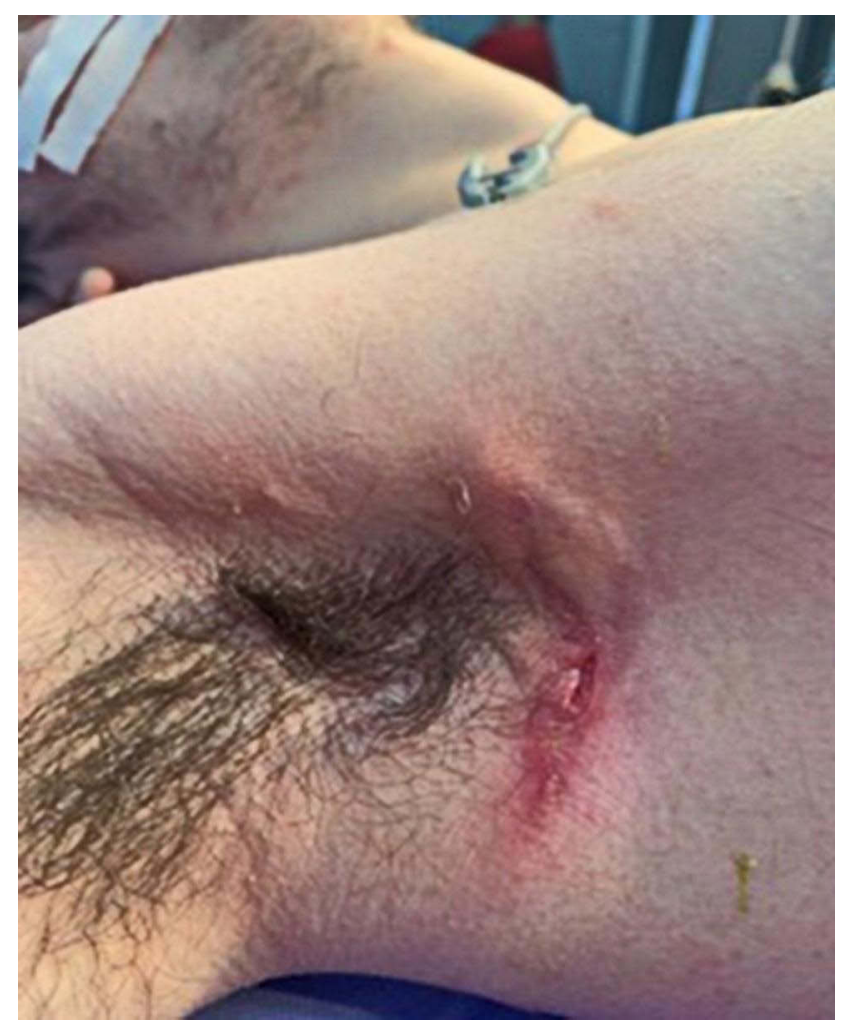

Figure 7 Bilateral axillary hidradenitis, Hurley stage II.

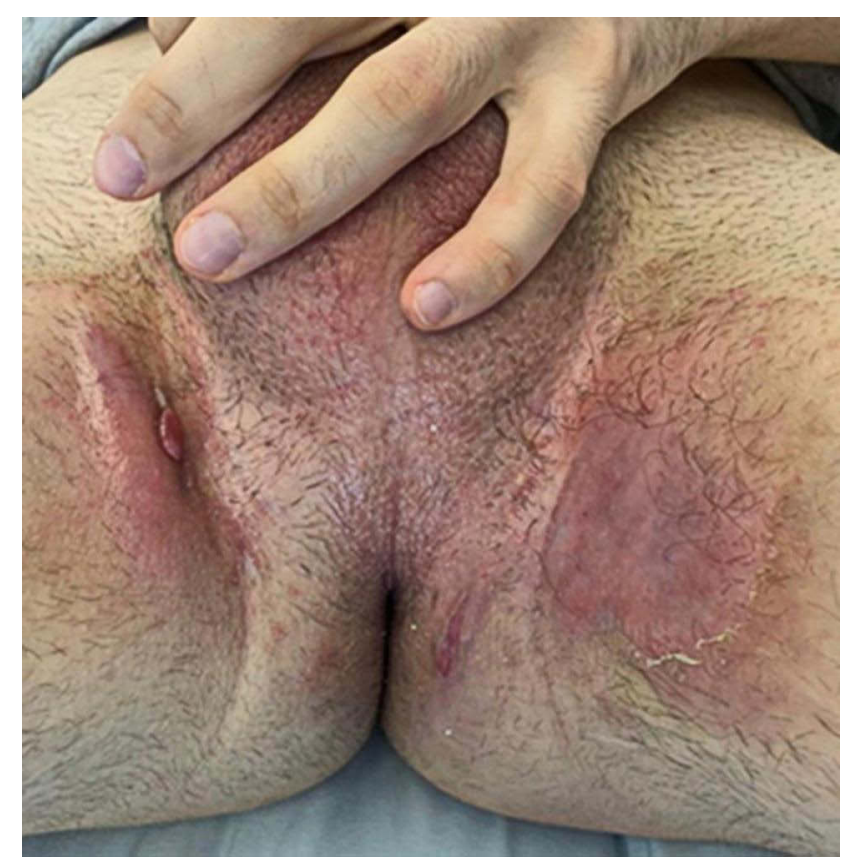

Figure 8 Suppurative hidradenitis in the groin and perineal.

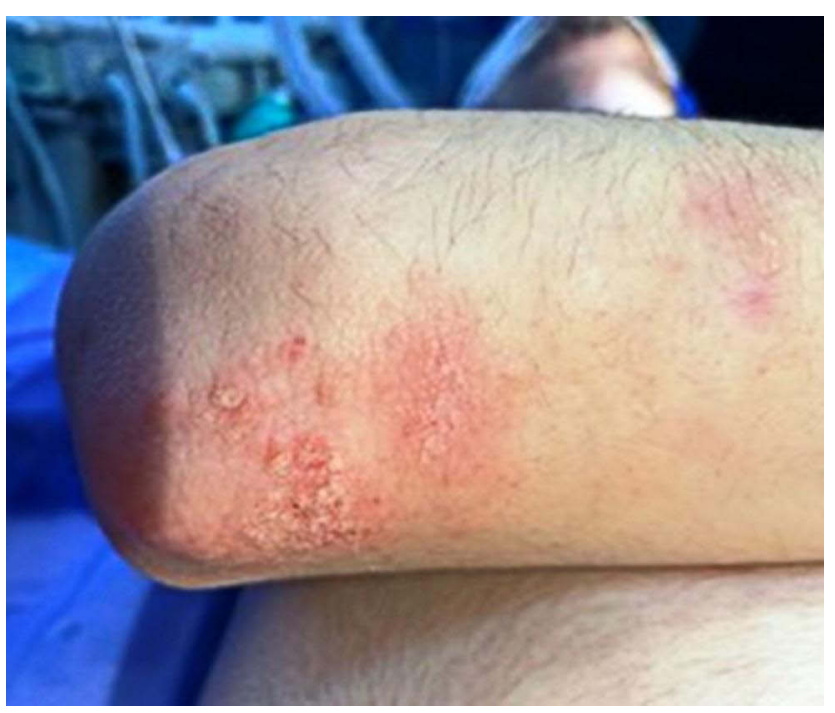

Figure 9 Psoriasis lesions on the elbows and back.

isolated from purulent axillary and perineal secretions. Given the recurrent nature of bilateral and inguinal axillary skin lesions, as well as associated conditions, the patient is diagnosed with Hurley stage II hidradenitis suppurativa or moderate disease according to IHS4 staging. During the hospitalization, the patient benefits from psychological counseling and psychological treatment for low self-esteem and anxiety, antibiotic therapy with Clindamycin according to the culture, incision and drainage of perineal abscesses, curettage of right axillary lesions, excision of left axillary 


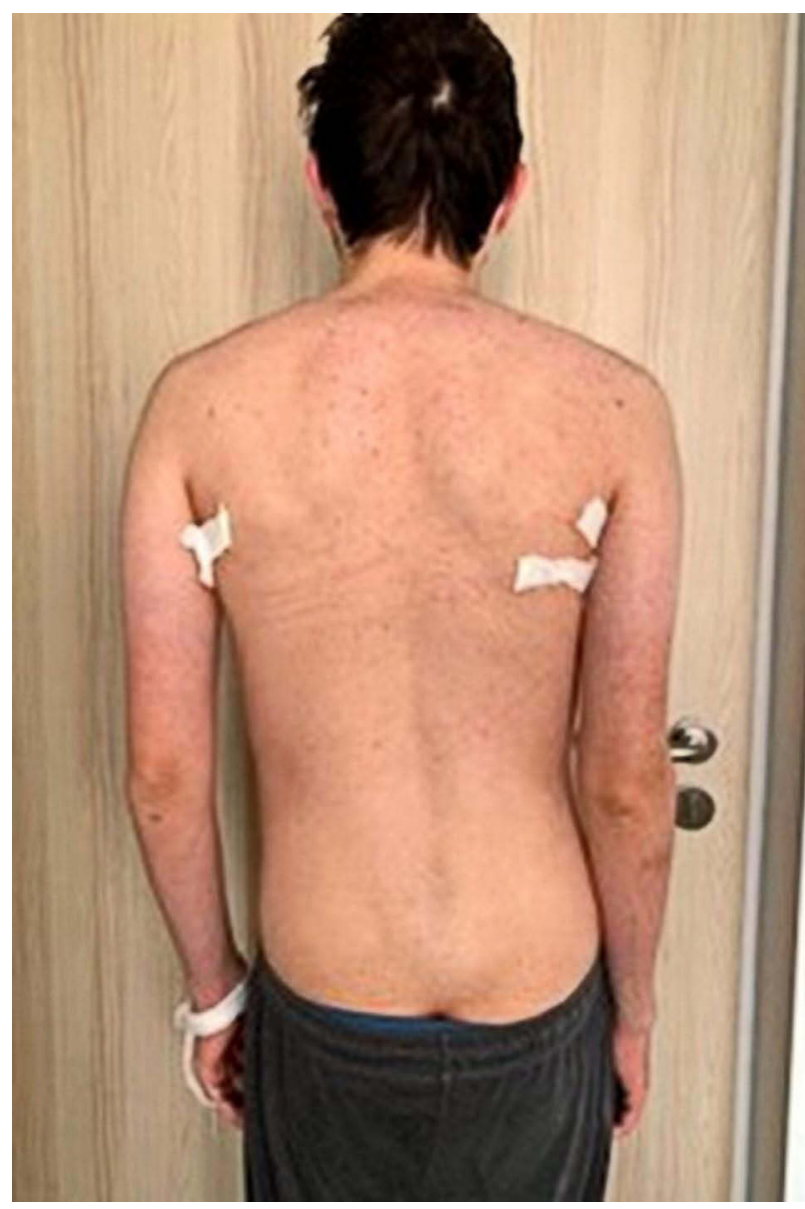

Figure 10 Dextroconvex dorsolumbar scoliosis and L5 spondylolysis.

lesions, with favorable evolution. Upon discharge, he was directed to the dermatology clinic to assess the appropriateness of treatment with biological agents, given the association with psoriasis. The pathological examination of the excised lesions surprises the skin with thickened epidermis; areas with inflammatory polymorphic infiltrate with suppurative foci; relatively numerous eosinophils throughout the dermis; aspects of obliterative endarteritis and ducts with rich inflammatory infiltrate with intraepithelial polymorphonuclear (Figures 11 and 12). The consent of the mother but also of the patient was obtained for the publication of the photos and the use of the data from the observation file.

\section{Sources of Information}

Using the Medical Subject Headings MeSH term "hidradenitis suppurativa", "children" and "Hurley disease", we performed a PubMed literature search for randomized controlled trials (RCTs), systematic reviews, observational studies, case series, and case reports from the earliest possible date to

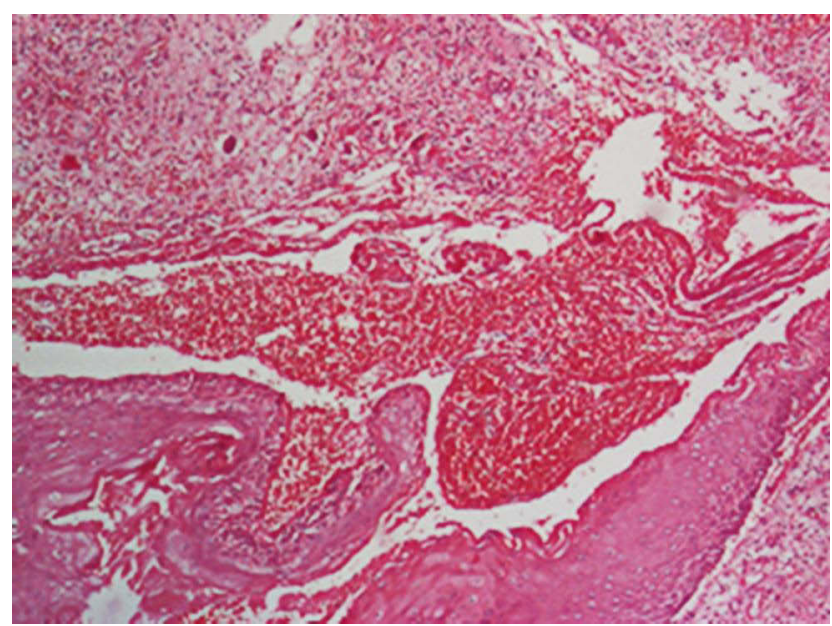

Figure II Thickened epidermis, edema, hemorrhagic exudate, inflammatory polymorphic infiltrate (HE stain, $\mathrm{x} 40$ ).

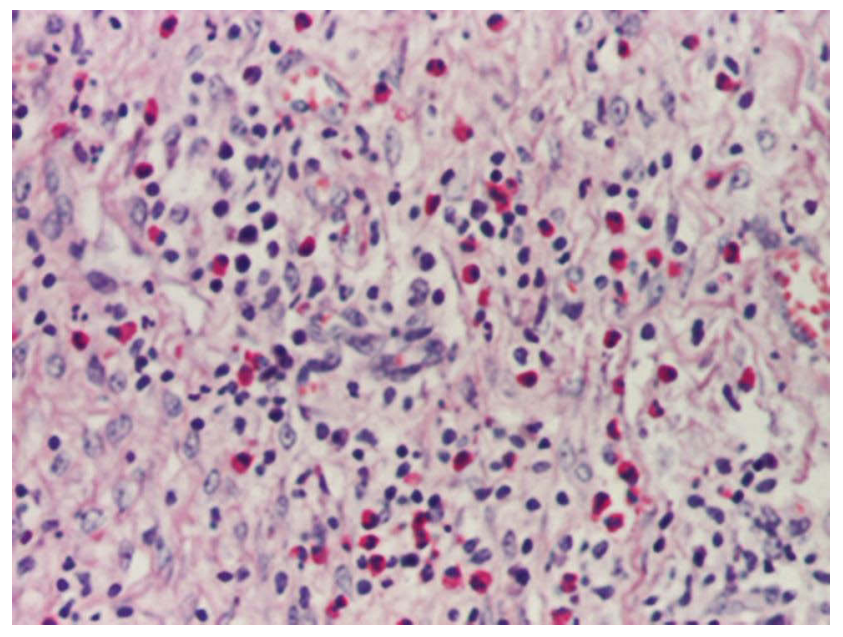

Figure 12 Areas with inflammatory polymorphic infiltrate, with relatively numerous eosinophils throughout the dermis (HE stain, $x \mid 00$ ).

February 2021. We included articles published in English and also searched the reference lists for other relevant articles. Searching the PubMed for term "hidradenitis suppurativa", 3348 articles were found, but when we added the term "children", only 191 articles returned. We have reviewed the title and the abstract of these articles and only 91 of them were identified as effectively analyzing pediatric cases and meeting inclusion criteria. We checked the full-text of these articles and extracted data on incidence, sex ratio, diagnosis, etiology, staging, associated abnormalities and treatment. We quoted 35 of them.

\section{Etiopathology}

The causes of this condition are not fully known, the disease being probably multifactorial. Up to $40 \%$ of 
patients have a positive family history, suggesting a genetic involvement, most likely with autosomal dominant transmission. ${ }^{5,10}$ Hormonal factors certainly have a role in the onset of the disease, since high frequencies of occurrence are in the postpubertal and premenopausal period. In addition, androgen-containing drugs precipitate the appearance or worsen the situation, and antiandrogenic medication has beneficial effects on suppurative hidradenitis. ${ }^{5,11}$ Obesity is a condition associated with this disease and seems to be aggravated by the metabolic syndrome, but also smoking, which increases 9.4 times the risk of developing this condition. ${ }^{2,12,13}$ Although the disease is classically associated with sweat gland damage, recent studies show that it is actually a condition of the follicular epithelium resulting from repetitive mechanical stress in genetically susceptible individuals. ${ }^{14}$ Thus, the occlusion of the pilosebaceous units leads to inflammation and the appearance of abscesses, and the subsequent rupture of the follicles leads to the appearance of fistulas that will become secondary. ${ }^{5,14}$ Clinically, edema, erythema, burning or pain and hyperhidrosis appear first; subsequently, the initially formed nodules join and form abscesses that rupture, eliminating foul-smelling secretions and eventually forming dermal sinuses, scars or comedones. The most commonly affected areas are the armpits, groin and perineal region, submammary area, and less often the neck area, pelvic girdle area or retroauricular area, as in one of the cases presented.

\section{Diagnosis, Staging and Associated Abnormalities}

The diagnosis of this condition is clinical, highlighting the typical lesions at various evolutionary stages and also anamnestic (chronic and recurrent); family investigation and genetic consultation should be part of the protocol. Biological investigations will detect a possibly associated anemia, the presence of inflammatory syndrome, and the germs responsible for the local infection to establish targeted antibiotic therapy. Risk factors such as smoking, obesity, female gender, family history, metabolic syndrome will be highlighted, as well as the association of other conditions such as irritable bowel syndrome, Crohn's disease, epithelial tumors, psoriasis, acne or spondyloarthropathy ${ }^{15}$ (as one of the cases presented). In a recent article, from a total of 153 HS cases in children, $34.0 \%$ had at least one somatic comorbidity compared with $4.9 \%$ of the controls. By the age of 23 years, $23.5 \%$ of the patients with HS and $8.7 \%$ of the
Table I Hurley Staging of Suppurative Hidradenitis

\begin{tabular}{|l|l|}
\hline $\begin{array}{l}\text { Hurley Clinical } \\
\text { Staging System }\end{array}$ & Characteristics \\
\hline Stage I (mild) & $\begin{array}{l}\text { Single or multiple abscesses without sinus } \\
\text { tracts and scarring }\end{array}$ \\
\hline Stage II (moderate) & $\begin{array}{l}\text { Recurrent abscesses with sinus tracts and } \\
\text { scarring; single or multiple widely separated } \\
\text { lesions }\end{array}$ \\
\hline Stage III (severe) & $\begin{array}{l}\text { Diffuse or multiple interconnected sinus } \\
\text { tracts and abscesses across the entire area }\end{array}$ \\
\hline
\end{tabular}

controls had at least one identified psychiatric comorbidity. ${ }^{4}$ Physicians should monitor young patients with HS for both somatic and psychiatric comorbidities. After establishing the diagnosis of HS, additional biological and imaging investigations are necessary to detect associated diseases, their discovery being important for treatment in a multidisciplinary team.

The Hurley staging system, as described in Table 1, is commonly used for assessing HS severity. ${ }^{5,8,14}$ Most patients have stage I (7-69\%) or II (28-83\%) of the disease, and in stage III are found between $4 \%$ and $22 \%$ of patients. ${ }^{13}$ The Hurley staging system is used for the selection of the appropriate treatment modality: medical therapy for Hurley stage I, local surgery for Hurley stage II and wide surgical excision for Hurley stage III. ${ }^{16}$ Although the Hurley scoring system is a simple tool, it is nonquantitative and static. Several other clinical measures for assessing HS disease severity were described, including the recently proposed HS Severity Score System (IHS4) and the Hidradenitis Suppurativa Clinical Response. ${ }^{17}$ IHS4 score is arrived at by the number of nodules (multiplied by 1) plus the number of abscesses (multiplied by 2) plus the number of drainage tunnels (multiplied by 4 ). A total score of 3 or less signifies mild, 4-10 signifies moderate and 11 or higher signifies severe disease. ${ }^{17}$

\section{Treatment}

The condition is chronic, recurrent and difficult to treat, so prevention measures have a special role. Unfortunately, there are currently no pediatric treatment guidelines; ${ }^{3}$ psychological counseling is very important in the case of adolescents, the patient and family must be aware of the lack of curative treatment, of the fact that the condition is not contagious and is not caused by poor hygiene. The treatment will be personalized and performed according to 
the stage of evolution, and the approach will be a multidisciplinary one (pediatric or general surgeon, plastic surgeon, dermatologist, psychotherapist, cardiologist, and a pediatrician specialized in nutrition diseases, an ophthalmologist or a neurosurgeon, as in our cases). Regardless of the stage of the disease, patients must adopt a healthy lifestyle, quit smoking, lose weight, eat healthy foods, and wear light cotton underwear, avoiding mechanical stress on the skin of vulnerable areas. ${ }^{18}$ Once signs of inflammation appear, washing with antibacterial soap, antiseptic solutions and applying warm compresses can stop the progression of the lesions. ${ }^{14}$ The quality of life of these patients is low, one of the reasons being pain, so analgesic treatment is important. ${ }^{19}$ Among the drugs used, and which have been shown to have a favorable effect, we mention resorcinol, a keratolytic and antiinflammatory agent, which is applied topically, in the form of a $15 \%$ solution, twice a day in exacerbations and once a day as maintenance, has the effect of reducing pain and promoting healing. ${ }^{20}$

As an antibiotic treatment, clindamycin can be used in all stages of development, both as a topical treatment (gel or $1 \%$ solution two to three times a day) and as a systemic treatment in stages II and III. For stages I and II, topically applied clindamycin may be as effective as oral tetracycline, the latter being the only antibiotic shown to have a favorable effect in oral administration, $500 \mathrm{mg}$ twice daily for four months. ${ }^{21}$ In severe cases, a combination of oral clindamycin $300 \mathrm{mg}$ twice daily and rifampicin $600 \mathrm{mg}$ daily for 10 weeks was shown to be effective. ${ }^{21}$ Unfortunately, with the discontinuation of oral antibiotic therapy, the recurrence rate increases. ${ }^{21}$ Hormone, antiandrogenic, or cyproterone acetate $100 \mathrm{mg}$ daily, finasteride $5 \mathrm{mg}$ daily, or norgestrel birth control pills for six months have been shown to be effective in some studies. ${ }^{5,11}$ Other treatments such as immunosuppressive, general or local (intralesional injections with triamcinolone acetate), treatment with biological agents (infliximab, adalimumab), or treatment with oral retinoids for one year (with immunomodulatory and antiproliferative effect) are recommended only selectively, at cases resistant to conventional treatment due to side effects. ${ }^{3,5,22}$ In a recent article, Hendricks et al tried to make a comparison between international guidelines for the management of HS and reached to the conclusion that variations exist across international treatment for this disease, and much collaboration is needed to put forth unified and updated recommendations. ${ }^{18}$ In Table 2, we summarized the most used forms of treatment for HS, according to the newest recommendations of the European and American Guidelines. ${ }^{20,23,24}$

Surgical treatment will be reserved for stage II cases with collected abscesses and will ensure the curettage of lesions and drainage of purulent collections; for stage III, it will ensure the removal of dermal sinuses or excision of superinfected tissues, and the revision of scars. Possible surgical interventions included limited excision with primary closure with or without gentamicin implant, deroofing of sinus tracts, wide excision closed by a musculocutaneous flap or by a split skin graft, wide excision closed by a biosynthetic skin substitute, wide excision healed by secondary intention, with or without using negative pressure dressing, wide excision healed by secondary intention using silastic foam dressing, carbon dioxide laser excision, and ablation. ${ }^{25}$ Due to the lack of studies in large groups of patients or compared to medical treatment, surgical excision of the affected areas is recommended only for cases resistant to conservative treatment. ${ }^{5}$

\section{Discussion}

Although the maximum incidence of the disease is in the 2-3rd decade of life and the appearance in children is rare, the youngest patient reported in the literature is 6 years old. ${ }^{11}$ We reported two cases in adolescence, and although the disease apparently mainly affects females, both of our patients are boys. But as two recent articles show, boys are more likely to have prepubescent onset of hidradenitis suppurativa and girls to have postpubescent onset. This shift in sex distribution is unexplained, but the authors hypothesize that, whereas the role of ovarian hormones in the pathogenesis of HS may underlie much of adultonset disease, it is less important in prepubescent disease. $^{2,9}$

The condition is little known, especially among primary care and pediatricians, and therefore special cases are worth publishing. We chose to report these cases, both patients being diagnosed late; in the long run, the effects can be devastating, with significant impact on quality of life due to pain, inability to perform various physical activities and decreased self-esteem. Especially in adolescents and young adults, friendships and social integration in school and at work are affected, with these patients registering an increased rate of school absenteeism or absence from work. ${ }^{19,26}$ Patients with HS have a higher prevalence of depression, anxiety, and suicidal ideation, demonstrating the impact of HS on psychosocial wellbeing and QoL. ${ }^{15,27}$ Our first patient underwent multiple 


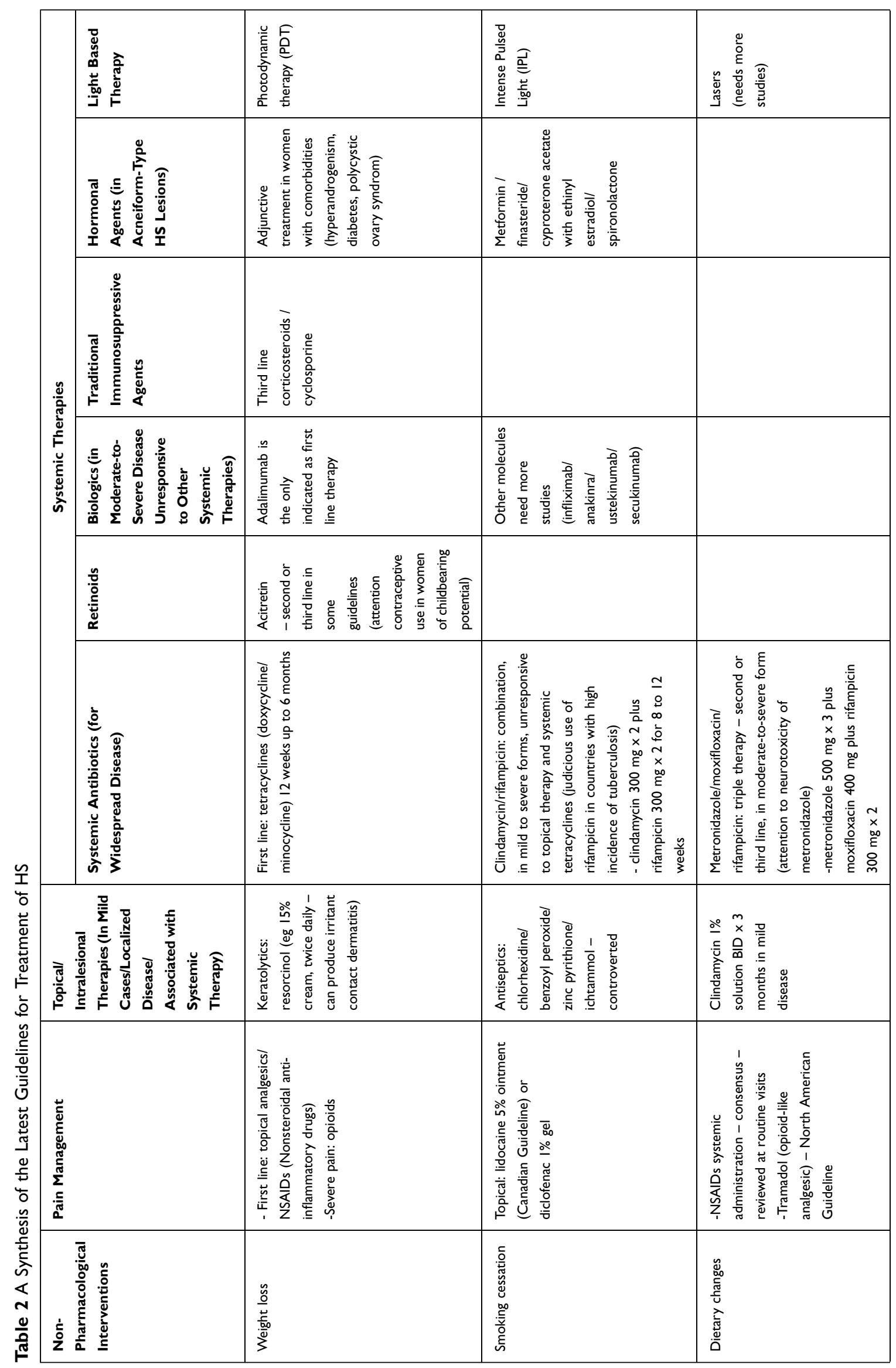




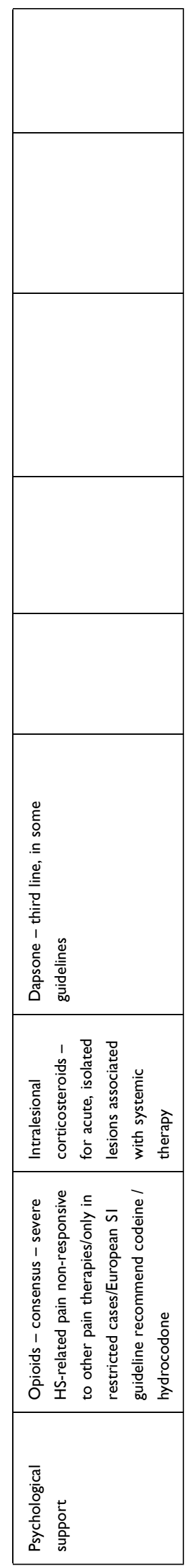

interventions, infected lesions not only responding to antibiotic treatmentbut also needed more hospitalizations and treatment with skin flaps; the last time was hospitalized for a continuous period of 3.5 months, time in which he suffered multiple mutilated surgeries and complications that demoralized him, reasons why he declared that he had suicidal intentions. The costs of patient hospitalization amounted 15,500 euro in total, which is important for east Europe, but the costs of hospitalization were fully covered by the state; the literature is also reporting increased costs of HS management compared to other skin conditionsfor example, psoriasis. ${ }^{28}$ Adults and children with HS had increased acute and chronic, cutaneous, extracutaneous and systemic infections, which were associated with increased mortality and cost in other hospitals too. ${ }^{29}$

Patient No. 1 underwent long-term surgery, with severe postoperative complications, and the rescue solution was excision of the affected areas (axillary and perineal) and grafting with free skin or advancing flaps; colostomy was needed to heal the perineal area. The literature also reports complications such as impaired limb mobility due to recurrent suppurations and scar tissue formation, rectal or urethral stenoses, and even squamous cell carcinomas, but unfortunately studies in large groups of patients on surgical treatment are lacking. ${ }^{5,30}$ Rarely, the localized infection can generalize, leading to sepsis, but more often superinfections occur, sometimes with hospital germs that are multidrug-resistant, as in our case. We obtained good results after the surgical treatment of excision-grafting of the axillary areas in the case of the first patient, the condition being thus limited (Figure 1). The literature reports that surgical interventions involve either the limited excision or radical wide excision of an involved region, and radical wide excision can be effective. ${ }^{24,31}$ Negative pressure wound therapy is an effective adjunct in wound healing and closure in the pediatric population. ${ }^{32}$ Although surgery is not without its challenges, it presents a safe option for pediatric HS patients with extensive scar or sinus tract formation. ${ }^{33}$ Scarring lesions generally require surgery. ${ }^{1}$

The second case presented is the typical example of an adolescent patient, introvert and anxious, embarrassed, who hid for several months the affection towards his parents. At the time of hospitalization in our clinic, the clinicalparaclinical investigations also revealed the presence of associated diseases, such as osteo-articular, cardiac, ocular and dermatological. Psychotherapy for low self-esteem, stress, anger, shame, depression and anxiety, topical 
treatment with antiseptic solutions, antibiotic therapy with clindamycin, excision and drainage of purulent collections led to a favorable evolution, and treatment with adalimumab remained in question, considering the association with psoriasis. The association of psoriasis, acne and osteoarticular diseases requires a careful follow-up of this adolescent from a psychosocial point of view, depression, anxiety and isolation being common in cases like this. ${ }^{15,27}$ Some precarious socio-economic conditions and an inadequate family or school environment can worsen the situation for children and adolescents because they are very easily influenced; therefore, the problem can sometimes take social aspects. ${ }^{34}$

Given the chronic and recurrent nature of the condition, as well as associated conditions, patients need longterm follow-up, with each case addressing a personalized management in a multidisciplinary team. We would like to emphasize once more the multidisciplinary aspect of the treatment; the first patient benefited from a pediatric surgeon, who led the surgical treatment and performed the colostomy, a plastic surgeon that performed the flaps and skin grafts; the dermatologist dealt with the follow-up and topical treatment after discharge. The psychotherapist, the infectionist doctor, the pathologist and the pediatrician specialized in nutrition diseases also contributed a lot to the patient's management. The management of the second patient was also performed by a multidisciplinary team, which included the pediatric surgeon, the orthopedist (pectus excavatum, scoliosis and associated spondylolysis), and the dermatologist. The cardiologist, the psychotherapist, the ophthalmologist also gave consultations and made recommendations for the associated diseases.

Prevention measures, psychological counseling and therapy, removal of risk factors as much as possible and medical therapy are the basis of the treatment pyramid. Because these patients have a $50 \%$ higher risk of developing any form of cancer compared to the general population, $^{35}$ we recommend a general clinicalparaclinical evaluation every six months.

\section{Conclusions}

Hidradenitis suppurativa is a severe chronic condition underdiagnosed in children, with long-term negative effects on the patient's psychosocial status and quality of life. Recognition of typical, recurrent lesions, history of the disease and family investigation establishes the diagnosis. Psychotherapy, health education, the adoption of a healthy lifestyle, the application of local prevention measures, and long-term multidisciplinary medical treatment are components of a correct management. Extensive surgical treatment should be restricted only to severe cases because it may lead to complications, extend the hospitalization period and increase psychological problems in adolescents. Although the number of studies concerning HS is increasing, further investigation is warranted to facilitate early diagnosis in children and determine the best management.

\section{Data Sharing Statement}

The datasets used and/or analyzed during the current study are available from the corresponding authors on reasonable requests.

\section{Institutional Review Board Statement}

The research was approved by the Ethics Committee of "Saint Mary" Emergency Children's Hospital, Iași.

\section{Informed Consent Statement}

Informed consent to publish the data was obtained from all subjects involved in the study, and the parents.

\section{Author Contributions}

All authors contributed to data analysis, drafting or revising the article, have agreed on the journal to which the article will be submitted, gave final approval for the version to be published, and agreed to be accountable for all aspects of the work. All authors have equal contributions to this work.

\section{Funding}

This research received no external funding.

\section{Disclosure}

The authors declare no conflicts of interest.

\section{References}

1. Mikkelsen PR, Jemec GB. Hidradenitis suppurativa in children and adolescents: a review of treatment options. Paediatr Drugs. 2014;16 (6):483-489. PMID: 25234961. doi:10.1007/s40272-014-0091-3

2. Vaiopoulos AG, Nikolakis G, Zouboulis CC. Hidradenitis suppurativa in paediatric patients: a retrospective monocentric study in Germany and review of the literature. $J$ Eur Acad Dermatol Venereol. 2020;34 (9):2140-2146. PMID: 32324936. doi:10.1111/jdv.16520

3. Rundle CW, Price KN, Hogeling M, Hsiao JL, Shi VY. Recent advances in hidradenitis suppurativa in pediatrics. Dermatol Online J. 2020;26(12):13030/qt5gt6m9n3. PMID: 33423411. doi:10.5070/ D32612051346 
4. Tiri H, Jokelainen J, Timonen M, Tasanen K, Huilaja L. Somatic and psychiatric comorbidities of hidradenitis suppurativa in children and adolescents. J Am Acad Dermatol. 2018;79(3):514-519. PMID: 29518461. doi:10.1016/j.jaad.2018.02.067

5. Lee EY, Alhusayen R, Lansang P, Shear N, Yeung J. What is hidradenitis suppurativa? Can Fam Physician. 2017;63(2):114-120. PMID: 28209676; PMCID: PMC5395382.

6. Mebazaa A, Ben Hadid R, Cheikh Rouhou R, et al. Hidradenitis suppurativa: a disease with male predominance in Tunisia. Acta Dermatovenerol Alp Pannonica Adriat. 2009;18(4):165-172.

7. Cosmatos I, Matcho A, Weinstein R, Montgomery MO, Stang P. Analysis of patient claims data to determine the prevalence of hidradenitis suppurativa in the United States. $J$ Am Acad Dermatol. 2013;68(3):412-419. doi:10.1016/j.jaad.2012.07.027

8. Canoui-Poitrine F, Le Thuaut A, Revuz JE, et al. Identification of three hidradenitis suppurativa phenotypes: latent class analysis of a cross-sectional study. J Invest Dermatol. 2013;133(6):1506-1511. doi: $10.1038 /$ jid.2012.472

9. Braunberger TL, Nicholson CL, Gold L, et al. Hidradenitis suppurativa in children: the Henry Ford experience. Pediatr Dermatol. 2018;35(3):370-373. PMID: 29575194. doi:10.1111/pde.13466

10. Al-Ali FM, Ratnamala U, Mehta TY, et al. Hidradenitis suppurativa (or acne inversa) with autosomal dominant inheritance is not linked to chromosome 1p21.1-1q25.3 region. Exp Dermatol. 2010;19 (9):851-853. doi:10.1111/j.1600-0625.2010.01088.x

11. Randhawa HK, Hamilton J, Pope E. Finasteride for the treatment of hidradenitis suppurativa in children and adolescents. JAMA Dermatol. 2013;149(6):732-735. doi:10.1001/jamadermatol.2013.2874

12. Vazquez BG, Alikhan A, Weaver AL, Wetter DA, Davis MD. Incidence of hidradenitis suppurativa and associated factors: a population-based study of Olmsted County, Minnesota. J Invest Dermatol. 2013;133(1):97-103. doi:10.1038/jid.2012.255

13. Sartorius K, Emtestam L, Jemec GB, et al. Objective scoring of hidradenitis suppurativa reflecting the role of tobacco smoking and obesity. $\mathrm{Br}$ J Dermatol. 2009;161:831-839. doi:10.1111/j.1365-2133.2009.09198.x

14. Alikhan A, Lynch PJ, Eisen DB. Hidradenitis suppurativa: a comprehensive review. $J$ Am Acad Dermatol. 2009;60 (4):539-561. doi:10.1016/j.jaad.2008.11.911

15. Pinter A, Kokolakis G, Rech J, et al. Hidradenitis suppurativa and concurrent psoriasis: comparison of epidemiology, comorbidity profiles, and risk factors. Dermatol Ther (Heidelb). 2020;10(4):721-734. doi:10.1007/s13555-020-00401-y

16. Ovadja ZN, Schuit MM, van der Horst CMAM, Lapid O. Inter- and intrarater reliability of Hurley staging for hidradenitis suppurativa. $\mathrm{Br}$ J Dermatol. 2019;181(2):344-349. doi:10.1111/bjd.17588

17. Zouboulis CC, Tzellos T, Kyrgidis A, et al. European hidradenitis suppurativa foundation investigator group. development and validation of the international hidradenitis suppurativa severity score system (IHS4), a novel dynamic scoring system to assess HS severity. Br J Dermatol. 2017;177(5):1401-1409. PMID: 28636793. doi: 10.1111/bjd.15748

18. Hendricks AJ, Hsiao JL, Lowes MA, Shi VY. A comparison of international management guidelines for hidradenitis suppurativa. Dermatology. 2021;237(1):81-96. PMID: 31645040. doi:10.1159/000503605

19. Dufour DN, Emtestam L, Jemec GB. Hidradenitis suppurativa: a common and burdensome, yet under-recognised, inflammatory skin disease. Postgrad Med J. 2014;90(1062):216-220. doi:10.1136/ postgradmedj-2013-131994
20. Zouboulis CC, Desai N, Emtestam L, et al. European S1 guideline for the treatment of hidradenitis suppurativa/acne inversa. J Eur Acad Dermatol Venereol. 2015;29(4):619-644. doi:10.1111/jdv.12966

21. Jemec GB, Wendelboe P. Topical clindamycin versus systemic tetracycline in the treatment of hidradenitis suppurativa. J Am Acad Dermatol. 1998;39(6):971-974. doi:10.1016/S0190-9622(98)70272-5

22. Kimball AB, Kerdel F, Adams D, et al. Adalimumab for the treatment of moderate to severe hidradenitis suppurativa: a parallel randomized trial. Ann Intern Med. 2012;157(12):846-855. doi:10.7326/00034819-157-12-201212180-00004

23. Ingram JR, Collier F, Brown D, et al. British association of dermatologists guidelines for the management of hidradenitis suppurativa (acne inversa) 2018. Br J Dermatol. 2019;180(5):1009-1017. doi:10.1111/bjd.17537

24. Alikhan A, Sayed C, Alavi A, et al. North American clinical management guidelines for hidradenitis suppurativa: a publication from the United States and Canadian hidradenitis suppurativa foundations: part ii: topical, intralesional, and systemic medical management. J Am Acad Dermatol. 2019;81(1):91-101. doi:10.1016/j.jaad.2019.02.068

25. Ingram JR, Woo PN, Chua SL, et al. Interventions for hidradenitis suppurativa. Cochrane Database Syst Rev. 2015;2015(10): CD010081. doi:10.1002/14651858.CD010081.pub2

26. Matusiak Ł, Bieniek A, Szepietowski JC. Hidradenitis suppurativa markedly decreases quality of life and professional activity. $J \mathrm{Am}$ Acad Dermatol. 2010;62:706-708. doi:10.1016/j.jaad.2009.09.021

27. Machado MO, Stergiopoulos V, Maes M, et al. Depression and anxiety in adults with hidradenitis suppurativa: a systematic review and meta-analysis. JAMA Dermatol. 2019;155(8):939-945. doi:10.10 01/jamadermatol.2019.0759

28. Kirby JS, Miller JJ, Adams DR, Leslie D. Health care utilization patterns and costs for patients with hidradenitis suppurativa. JAMA Dermatol. 2014;150(9):937-944. doi:10.1001/jamadermatol.2014.691

29. Lee HH, Patel KR, Singam V, Rastogi S, Silverberg JI. Associations of cutaneous and extracutaneous infections with hidradenitis suppurativa in U.S. children and adults. Br J Dermatol. 2020;182 (2):327-334. PMID: 31049925. doi:10.1111/bjd.18093

30. Dufresne RG Jr, Ratz JL, Bergfeld WF, Roenigk RK. Squamous cell carcinoma arising from the follicular occlusion triad. J Am Acad Dermatol. 1996;35(3 Pt 1):475-477. doi:10.1016/S0190-9622(96) 90632-5

31. Rambhatla PV, Lim HW, Hamzavi I. A systematic review of treatments for hidradenitis suppurativa. Arch Dermatol. 2012;148 (4):439-446. doi:10.1001/archdermatol.2011.1950

32. Rentea RM, Somers KK, Cassidy L, Enters J, Arca MJ. Negative pressure wound therapy in infants and children: a single-institution experience. J Surg Res. 2013;184(1):658-664. PMID: 23768766. doi:10.1016/j.jss.2013.05.056

33. Ge S, Ngaage LM, Orbay H, Silverman RP, Rasko YM, Rada EM. Surgical management of pediatric hidradenitis suppurativa: a case series and review of the literature. Ann Plast Surg. 2020;84(5):570-574. PMID: 31913903. doi:10.1097/SAP.0000000000002195

34. Țarcă E, Roșu ST, Cojocaru E, et al. Socio-epidemiological factors with negative impact on infant morbidity, mortality rates, and the occurrence of birth defects. Healthcare. 2021;9(4):384. doi:10.3390/ healthcare9040384

35. Lapins J, Ye W, Nyrén O, et al. Incidence of cancer among patients with hidradenitis suppurativa. Arch Dermatol. 2001;137:730-734. 


\section{Video abstract}

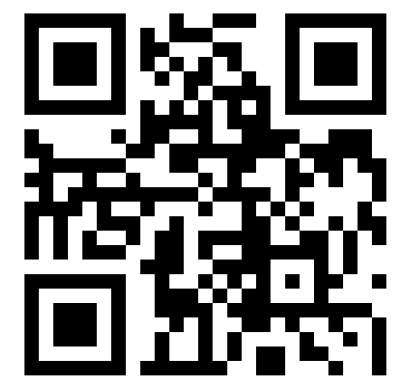

Point your SmartPhone at the code above. If you have a $Q R$ code reader, the video abstract will appear. Or use: https://youtu.be/00jselpGQUo

\section{Publish your work in this journal}

The Journal of Multidisciplinary Healthcare is an international, peerreviewed open-access journal that aims to represent and publish research in healthcare areas delivered by practitioners of different disciplines. This includes studies and reviews conducted by multidisciplinary teams as well as research which evaluates the results or conduct of such teams or healthcare processes in general. The journal covers a very wide range of areas and welcomes submissions from practitioners at all levels, from all over the world. The manuscript management system is completely online and includes a very quick and fair peer-review system. Visit http://www.dovepress.com/testimonials. php to read real quotes from published authors. 